\title{
GENERALIZED TEST IDEALS, SHARP F-PURITY, AND SHARP TEST ELEMENTS
}

\author{
KARL SCHWEDE
}

\begin{abstract}
Consider a pair $\left(R, \mathfrak{a}^{t}\right)$ where $R$ is a ring of positive characteristic, $\mathfrak{a}$ is an ideal such that $\mathfrak{a} \cap R^{\circ} \neq \emptyset$, and $t>0$ is a real number. In this situation we have the ideal $\tau_{R}\left(\mathfrak{a}^{t}\right)$, the generalized test ideal associated to $\left(R, \mathfrak{a}^{t}\right)$ as defined by Hara and Yoshida. We show that $\tau_{R}\left(\mathfrak{a}^{t}\right) \cap R^{\circ}$ is made up of appropriately defined generalized test elements which we call sharp test elements. We also define a variant of $F$-purity for pairs, sharp F-purity, which interacts well with sharp test elements and agrees with previously defined notions of $F$-purity in many common situations. We show that if $\left(R, \mathfrak{a}^{t}\right)$ is sharply $F$-pure, then $\tau_{R}\left(\mathfrak{a}^{t}\right)$ is a radical ideal. Furthermore, by following an argument of Vassilev, we show that if $R$ is a quotient of an $F$-finite regular local ring and $\left(R, \mathfrak{a}^{t}\right)$ is sharply $F$-pure, then $R / \tau_{R}\left(\mathfrak{a}^{t}\right)$ itself is $F$-pure. We conclude by showing that sharp $F$-purity can be used to define the $F$-pure threshold. As an application we show that the $F$-pure threshold must be a rational number under certain hypotheses.
\end{abstract}

\section{Introduction}

In this note, we consider the various aspects of the generalized test ideal $\tau_{R}\left(\mathfrak{a}^{t}\right)$ associated to the pair $\left(R, \mathfrak{a}^{t}\right)$, as defined by Hara and Yoshida [7]; also see [19]. Here $R$ is a ring of positive characteristic $p, \mathfrak{a}$ is an ideal such that $\mathfrak{a} \cap R^{\circ} \neq \emptyset$ and $t>0$ is a real number. The ideal $\tau_{R}\left(\mathfrak{a}^{t}\right)$ is a positive characteristic analogue of the multiplier ideal (see [17] and [7]) which has been heavily studied in recent years. The reason for the name test ideal is that in the classical case where $\mathfrak{a}=R, \tau\left(R^{1}\right) \cap R^{\circ}$ is equal to the set of tight closure test elements (elements of $R^{\circ}$ which can be used to "test" tight closure containments; see [9]).

However, when tight closure for pairs $\left(R, \mathfrak{a}^{t}\right)$ was defined, see [7] and also [19], a generalization of test elements, called $\mathfrak{a}^{t}$-test elements, was introduced and studied. In particular, it was easy to see that $\tau_{R}\left(\mathfrak{a}^{t}\right) \cap R^{\circ}$ is not equal to the set of these test elements even if $R$ is regular, $\mathfrak{a}$ is principal and $t=1$. We introduce a new type of generalized test element which we call a sharp test element. An important point is that these sharp test elements still "test" tight closure containments. Furthermore, $\tau_{R}\left(\mathfrak{a}^{t}\right) \cap R^{\circ}$ is in fact equal to the set of sharp test elements. Thus our main result is the following.

Corollary 2.8 The set of $\mathfrak{a}^{t}$-sharp test elements is equal to $\tau\left(\mathfrak{a}^{t}\right) \cap R^{\circ}$.

Received by the editors October 18, 2007.

2000 Mathematics Subject Classification. 13A35, 14 B05.

Key words and phrases. tight closure, F-pure, test ideal, test element.

The author was partially supported by a National Science Foundation postdoctoral fellowship. 
This definition of generalized test elements also suggests a redefinition of $F$-purity for pairs $\left(R, \mathfrak{a}^{t}\right)$, which we call sharp F-purity; compare with [6] and [18]. Once we have this definition, we are able to generalize several results from the classical case $(\mathfrak{a}=R)$, to the case of a pair $\left(R, \mathfrak{a}^{t}\right)$. In particular, simply following classical arguments, we are able to show that if $\left(R, \mathfrak{a}^{t}\right)$ is sharply $F$-pure, then $\tau_{R}\left(\mathfrak{a}^{t}\right)$ is a radical ideal; see Corollary 3.15. Furthermore, under certain mild hypotheses on $R$, we are able to obtain the following generalization of a theorem of Vassilev; see [21].

Corollary 4.3 Suppose $R$ is a quotient of an $F$-finite regular ring, $\mathfrak{a} \subseteq R$ an ideal such that $\mathfrak{a} \cap R^{\circ} \neq \emptyset$ and $t>0$ a real number such that $\left(R, \mathfrak{a}^{t}\right)$ is sharply F-pure. Then $R / \tau_{R}\left(\mathfrak{a}^{t}\right)$ is F-pure.

This corollary should be thought of as related to [12, Theorem 1], [1, Proposition $3.2]$, and [16, Theorem 5.5], all results in characteristic zero which use very different techniques.

Finally, we also show that sharp $F$-purity can be used to define c(a), the $F$-pure threshold of $\left(R, \mathfrak{a}^{*}\right)$; see [20]. As an easy corollary we are able to show that if $\left(R, \mathfrak{a}^{\mathrm{c}(\mathfrak{a})}\right)$ is sharply $F$-pure, then $\mathrm{c}(\mathfrak{a})$ is a rational number that can be written as a quotient whose denominator is not a multiple of $p=\operatorname{char} R$; see Corollary 5.4.

It should be noted that many of the arguments presented in this paper are simply generalizations of arguments from the "classical case". However, this strongly suggests that the sharp test element which we introduce is the correct notion of a generalized test element and that sharp F-purity is the right notion of $F$-purity for pairs.

\section{Sharp Test Elements}

All rings will be assumed to be Noetherian excellent reduced and containing a field of characteristic $p>0$. Given a ring $R$, we define $R^{\circ}$ to be the set of all elements of $R$ not contained in any minimal prime of $R$. If $\mathfrak{a}$ is an ideal of $R$ such that $\mathfrak{a} \cap R \neq \emptyset$, then we define $\mathfrak{a}^{0}=R$. If $I$ is an ideal of $R$, then we define $I^{\left[p^{e}\right]}$ to be the ideal generated by the $p^{e}$ th powers of elements of $I$.

Definition 2.1. [7] [19] [9] Let $R$ be a reduced ring and let $I$ be an ideal of $R$. Further suppose that $\mathfrak{a}$ is an ideal such that $\mathfrak{a} \cap R^{\circ} \neq \emptyset$ and $t>0$ is a real number. We say that $z$ is in the $\mathfrak{a}^{t}$-tight closure of $I$, denoted $I^{* \mathfrak{a}^{t}}$, if there exists a $c \in R^{\circ}$ such that

$$
c \mathfrak{a}^{\left\lceil t p^{e}\right\rceil} z^{p^{e}} \subseteq I^{\left[p^{e}\right]}
$$

for all $e \gg 0$.

The next two lemmas show that we can alter certain conditions in the definition of $I^{* \mathfrak{a}^{t}}$ without changing $I^{* \mathfrak{a}^{t}}$.

Lemma 2.2. Assume the notation of Definition 2.1. Then $z \in I^{* \mathfrak{a}^{t}}$ if and only if there exists some $c \in R^{\circ}$ such that

$$
c \mathfrak{a}^{\left\lceil t p^{e}\right\rceil} z^{p^{e}} \subseteq I^{\left[p^{e}\right]}
$$

for all $e \geq 0$. 
Proof. We follow the main idea of the proof of [9, Proposition 4.1(c)]. Suppose that there exists a $c^{\prime}$ such that $c^{\prime} \mathfrak{a}^{\left\lceil t p^{e}\right\rceil} z^{p^{e}} \subseteq I^{\left[p^{e}\right]}$ for all $e>e_{0}$. Since $R$ is reduced, we note that $S=\left(R^{\circ}\right)^{-1} R$ is a product of fields, and thus every ideal in $S$ is radical. Therefore $I^{[q]} S=I S$ for all $q=p^{e}$. Also note that since $\mathfrak{a} \cap R^{\circ} \neq \emptyset$ we have $\mathfrak{a}^{\lceil t q]} S=S$. But now if $c^{\prime} \mathfrak{a}^{\left[t p^{e}\right\rceil} z^{p^{e}} \subseteq I^{\left[p^{e}\right]}$ for $e>e_{0}$, we see that $z \in I S$ and furthermore that $z^{p^{e}} \in(I S)^{\left[p^{e}\right]}=I^{\left[p^{e}\right]} S$ for all $e$, and in particular, for each $e \leq e_{0}$. Thus we can pick $c_{e} \in R^{\circ}$ so that $c_{e} z^{p^{e}} \in I^{\left[p^{e}\right]}$ for each $e \leq e_{0}$. We set $c=c^{\prime} \prod_{i=1, \ldots, e_{0}} c_{i}$. It follows easily that $c \mathfrak{a}^{\left[t p^{e}\right\rceil} z^{p^{e}} \subseteq I^{\left[p^{e}\right]}$ for all $e \geq 0$.

Lemma 2.3. Assume the notation of Definition 2.1. Then $z \in I^{* \mathfrak{a}^{t}}$ if and only if there exists some $c \in R^{\circ}$ such that

$$
c \mathfrak{a}^{\left\lceil t\left(p^{e}-1\right)\right\rceil} z^{p^{e}} \subseteq I^{\left[p^{e}\right]} .
$$

for all $e \geq 0$.

Proof. The idea of the proof is essentially the same as in [6, Proposition 2.2]. Suppose that there exists a $c \in R^{\circ}$ such that $c \mathfrak{a}^{\left[t p^{e}\right\rceil} z^{p^{e}} \in I^{\left[p^{e}\right]}$ for all $e \geq 0$. Let $n$ be an exponent such that $\mathfrak{a}^{n} \mathfrak{a}^{\left\lceil t\left(p^{e}-1\right)\right\rceil} \subseteq\left(\mathfrak{a}^{\left\lceil t p^{e}\right\rceil}\right)$ for all $e \geq 0$ (for example, $n=\lceil t+1\rceil$ works). Choose $f \in \mathfrak{a} \cap R^{\circ}$. Then note that $\left(c f^{n}\right) \mathfrak{a}^{\left\lceil t\left(p^{e}-1\right)\right\rceil} z^{p^{e}} \subseteq\left(c \mathfrak{a}^{n}\right) \mathfrak{a}^{\left\lceil t\left(p^{e}-1\right)\right\rceil} z^{p^{e}} \subseteq$ $I^{\left[p^{e}\right]}$ for all $e \geq 0$.

It is the characterization of $I^{* \mathfrak{a}^{t}}$ from Lemma 2.3 that we will make use of for the remainder of the paper.

We now remind the reader of the definition of the generalized test ideal.

Definition 2.4. [7] Assume the notation of 2.1. Recall that the generalized test ideal of $\mathfrak{a}^{t}$, denoted by $\tau\left(\mathfrak{a}^{t}\right)$ is simply defined to be

$$
\bigcap_{J \subseteq R}\left(J: J^{* \mathfrak{a}^{t}}\right) \text { where } J \text { runs through all ideals of } R \text {. }
$$

We next define a new type of test element, the sharp test element.

Definition 2.5. We define $c \in R$ to be an $\mathfrak{a}^{t}$-sharp test multiplier for $R$ if, for all ideals $I$ and all $z \in I^{* \mathfrak{a}^{t}}$, we have $c \mathfrak{a}^{\left[t\left(p^{e}-1\right)\right]} z^{p^{e}} \subseteq I^{\left[p^{e}\right]}$ for all $e \geq 0$. We say that $c \in R^{\circ}$ is an $\mathfrak{a}^{t}$-sharp test element if $c$ is also an $\mathfrak{a}^{t}$-sharp test multiplier.

Notice first that if $c$ is an $\mathfrak{a}^{t}$-sharp test multiplier, then $c$ is in $\tau\left(\mathfrak{a}^{t}\right)$ because we can always consider the situation where $e=0$. We will show that the reverse inclusion also holds. First we need a lemma.

Lemma 2.6. Suppose that $z \in I^{* \mathfrak{a}^{t}}$. Then for every power $q=p^{e}, e \geq 0$ we have $\mathfrak{a}^{\lceil t(q-1)\rceil} z^{q} \subseteq\left(I^{[q]}\right)^{* \mathfrak{a}^{t}}$.

Proof. By assumption, we know that there exists a $c \in R^{\circ}$ such that $c a^{\left\lceil t\left(p^{d}-1\right)\right\rceil} z^{p^{d}} \subseteq$ $I^{\left[p^{d}\right]}$ for all $d \geq 0$. For every $f \in \mathfrak{a}^{[t(q-1)\rceil}$, we wish to show that there exists a $c^{\prime} \in R^{\circ}$ such that for all $d \geq 0$,

$$
c^{\prime} \mathfrak{a}^{\left\lceil t\left(p^{d}-1\right)\right\rceil}\left(f z^{q}\right)^{p^{d}} \subseteq\left(I^{[q]}\right)^{\left[p^{d}\right]}=I^{\left[p^{e+d}\right]} .
$$

But note that $\mathfrak{a}^{\left\lceil t\left(p^{d}-1\right)\right\rceil}\left(f z^{q}\right)^{p^{d}} \subseteq \mathfrak{a}^{\left\lceil t\left(p^{d}-1\right)\right\rceil}\left(\mathfrak{a}^{[t(q-1)\rceil} z^{q}\right)^{p^{d}}$ and so it is enough to show that

$$
c^{\prime} \mathfrak{a}^{\left[t\left(p^{d}-1\right)\right\rceil}\left(\mathfrak{a}^{[t(q-1)\rceil} z^{q}\right)^{p^{d}} \subseteq\left(I^{[q]}\right)^{\left[p^{d}\right]}=I^{\left[p^{e+d}\right]} .
$$


Now we observe that $\mathfrak{a}^{\left\lceil t\left(p^{d}-1\right)\right\rceil}\left(\mathfrak{a}^{\lceil t(q-1)\rceil} z^{q}\right)^{p^{d}}=\mathfrak{a}^{\left(\left\lceil t\left(p^{d}-1\right)\right\rceil+p^{d}\left\lceil t\left(p^{e}-1\right)\right\rceil\right)} z^{p^{e+d}}$. Set $c^{\prime}=$ $c$, and we see it is sufficient to check that $\left\lceil t\left(p^{d}-1\right)\right\rceil+p^{d}\left\lceil t\left(p^{e}-1\right)\right\rceil \geq\left\lceil t\left(p^{e+d}-1\right)\right\rceil$ for all $d \geq 0$. However, it is easy to see that

$\left\lceil t\left(p^{d}-1\right)\right\rceil+p^{d}\left\lceil t\left(p^{e}-1\right)\right\rceil \geq t\left(p^{d}-1\right)+p^{d} t\left(p^{e}-1\right)=t\left(p^{d}-1+p^{d+e}-p^{d}\right)=t\left(p^{d+e}-1\right)$

But the left side is an integer, and our needed inequality is proven.

Theorem 2.7. The set of $\mathfrak{a}^{t}$-sharp test multipliers is equal to $\tau\left(\mathfrak{a}^{t}\right)$.

Proof. Suppose that $c \in \tau\left(\mathfrak{a}^{t}\right)$ and suppose $z \in I^{* \mathfrak{a}^{t}}$. But then, using Lemma 2.6, for every $e \geq 0$ with $q=p^{e}$ we have,

$$
\mathfrak{a}^{\lceil t(q-1)\rceil} z^{q} \subseteq\left(I^{[q]}\right)^{* \mathfrak{a}^{t}}
$$

and so

$$
c \mathfrak{a}^{\lceil t(q-1)\rceil} z^{q} \subseteq I^{[q]}
$$

which proves that $c$ is an $\mathfrak{a}^{t}$-sharp test multiplier.

Corollary 2.8. The set of $\mathfrak{a}^{t}$-sharp test elements is equal to $\tau\left(\mathfrak{a}^{t}\right) \cap R^{\circ}$.

We can also show that sharp test elements exist.

Theorem 2.9. Suppose we are given a reduced $F$-finite ring $R$, an ideal $\mathfrak{a} \subseteq R$ such that $\mathfrak{a} \cap R^{\circ} \neq \emptyset$ and a real number $t>0$. Then $\mathfrak{a}^{t}$-sharp test elements exist; in other words, $\tau\left(\mathfrak{a}^{t}\right) \cap R^{\circ} \neq \emptyset$.

Proof. By hypothesis, $R$ is $F$-finite, reduced and $\mathfrak{a} \cap R^{\circ} \neq \emptyset$. Thus it follows from [7, Theorem 6.4] that there exists some element $c \in R^{\circ}$ such that for all ideals $I$, all $z \in I^{* \mathfrak{a}^{t}}$ and all $e \geq 0$, we have $c \mathfrak{a}^{\left\lceil t p^{e}\right\rceil} z^{p^{e}} \subseteq I^{\left[p^{e}\right]}$. Choose an element $d \in R^{\circ}$ such that $d \mathfrak{a}^{\left\lceil t\left(p^{e}-1\right)\right\rceil} \subseteq \mathfrak{a}^{\left\lceil t p^{e}\right\rceil}$ for all $e \geq 0$ (note that such an element exists since $\left.\mathfrak{a} \cap R^{\circ} \neq \emptyset\right)$. Then $c^{\prime}=c d \in R^{\circ}$ is a sharp test element since

$$
c^{\prime} \mathfrak{a}^{\left\lceil t\left(p^{e}-1\right)\right\rceil} z^{p^{e}}=c d \mathfrak{a}^{\left\lceil t\left(p^{e}-1\right)\right\rceil} z^{p^{e}} \subseteq c \mathfrak{a}^{\left\lceil t p^{e}\right\rceil} z^{p^{e}} \subseteq I^{\left[p^{e}\right]} .
$$

See [5, Section 2] for a comparison of a different sort of test element with elements of the generalized test ideal.

\section{Sharp F-purity}

In this section we define and study a variant of $F$-purity for pairs that works well with these sharp test elements. We use the following notation throughout this section. Let $R$ be an $F$-finite reduced ring of positive characteristic $p>0$. Further suppose that $\mathfrak{a}$ is an ideal with $\mathfrak{a} \cap R^{\circ} \neq \emptyset$ and $t>0$ is a real number. If $\mathfrak{a}=(f)$ is a principal ideal, then we will write $\left(R, f^{t}\right)$ for the pair $\left(R, \mathfrak{a}^{t}\right)$.

Recall that a pair $\left(R, \mathfrak{a}^{t}\right)$ is said to be $F$-pure if for every $e \gg 0$ there exists a $f \in \mathfrak{a}^{\left\lfloor t\left(p^{e}-1\right)\right\rfloor}$, such that the map

$$
f^{\frac{1}{p^{e}}} F^{e}: R \rightarrow R^{\frac{1}{p^{e}}},
$$

that sends 1 to $f^{\frac{1}{p^{e}}}$, splits. Recall that $R$ itself is said to be $F$-pure if $\left(R, R^{1}\right)$ is $F$-pure. See [18, Definition 3.1] and compare with [6, Section 2] and [10]. Also recall that a pair $\left(R, \mathfrak{a}^{t}\right)$ is said to be strongly $F$-pure if there exists $e>0$ (equivalently 
for all $e \gg 0$ ) and $f \in \mathfrak{a}^{\left\lceil t p^{e}\right\rceil}$ such that the map $f^{\frac{1}{p^{e}}} F^{e}$ splits; see [20]. Note that a strongly $F$-pure pair is $F$-pure; see [20, Proposition 1.5].

Definition 3.1. We say that $R$ is $\mathfrak{a}^{t}$-sharply $F$-pure, or that the pair $\left(R, \mathfrak{a}^{t}\right)$ is sharply $F$-pure, if there exist infinitely many integers $e \geq 0$ and associated elements $f_{e} \in \mathfrak{a}^{\left\lceil t\left(p^{e}-1\right)\right\rceil}$ such that each map

$$
f_{e}^{\frac{1}{p^{e}}} F^{e}: R \rightarrow R^{\frac{1}{p^{e}}}
$$

which sends 1 to $f_{e}^{\frac{1}{p^{e}}}$, splits.

Remark 3.2. It is easy to see that if $\left(R, \mathfrak{a}^{t}\right)$ is $F$-pure in the usual sense and $\left(p^{e}-1\right) t$ is an integer for infinitely many $e>0$ (equivalently, $\left(p^{e}-1\right) t$ is an integer for some $e>0$, equivalently $t$ is a rational number which can be written with an integer denominator which is not a multiple of $p)$, then $R$ is $\mathfrak{a}^{t}$-sharply $F$-pure. Furthermore, if $\left(R, \mathfrak{a}^{t}\right)$ is strongly $F$-pure, it is clearly both sharply $F$-pure and $F$-pure.

It turns out that one only needs to exhibit a single splitting in order to prove that a pair is sharply $F$-pure, as the following proposition shows.

Proposition 3.3. Suppose that there exists a single $e>0$ and an element $f \in$ $\mathfrak{a}^{\left\lceil t\left(p^{e}-1\right)\right\rceil}$ such that the map $f^{\frac{1}{p^{e}}} F^{e}: R \rightarrow R^{\frac{1}{p^{e}}}$ splits. Then $\left(R, \mathfrak{a}^{t}\right)$ is sharply F-pure.

Proof. It is sufficient to show that for all $d=n e, n$ a positive integer, there exists an element $g \in \mathfrak{a}^{\left\lceil t\left(p^{d}-1\right)\right\rceil}$ such that $g^{\frac{1}{p^{d}}} F^{d}: R \rightarrow R^{\frac{1}{p^{d}}}$ splits. By using the identification of $R^{\frac{1}{p^{e}}}$ with $R$ (since $R$ is reduced) we compose the map $f^{\frac{1}{p^{e}}} F^{e}$ with itself $n$-times. This is a map from $R$ to $R^{\frac{1}{p^{n e}}}$ which sends 1 to $f^{\frac{1+p^{e}+p^{2 e}+\ldots+p^{(n-1) e}}{p^{n e}}}$, and this map splits by construction. Thus, it is sufficient to show that $f^{1+p^{e}+\cdots+p^{(n-1) e}} \in \mathfrak{a}^{\left\lceil t\left(p^{n e}-1\right)\right\rceil}$. However, we do know that

$$
f^{1+\cdots+p^{(n-1) e}} \in \mathfrak{a}^{\left(1+\cdots+p^{(n-1) e}\right)\left\lceil t\left(p^{e}-1\right)\right\rceil} .
$$

Thus it is sufficient to show that

$$
\left(1+\cdots+p^{(n-1) e}\right)\left\lceil t\left(p^{e}-1\right)\right\rceil \geq\left\lceil t\left(p^{n e}-1\right)\right\rceil .
$$

But we do know that

$$
\left(1+\cdots+p^{(n-1) e}\right)\left\lceil t\left(p^{e}-1\right)\right\rceil \geq\left(1+\cdots+p^{(n-1) e}\right) t\left(p^{e}-1\right)=t\left(p^{n e}-1\right)
$$

Thus, since the left side is an integer, we are done.

Corollary 3.4. If there is an $f \in R$ such that the map $f^{\frac{1}{p^{e}}} F^{e}: R \rightarrow R^{\frac{1}{p^{e}}}$ splits, then $\left(R, f^{\frac{1}{p^{e}-1}}\right)$ is sharply F-pure.

We now show that if we are working with principal ideals, then $F$-purity and sharp $F$-purity agree much of the time.

Proposition 3.5. If $\left(R, f^{t}\right)$ is sharply F-pure, then $\left(R, f^{t}\right)$ is F-pure (and in fact the map for determining $F$-purity splits at every $e \geq 0$ ).

Proof. Choose $d>0$ and consider the map

$$
\left(f^{\left\lfloor t\left(p^{d}-1\right)\right\rfloor}\right)^{\frac{1}{p^{d}}} F^{d}: R \rightarrow R^{\frac{1}{p^{d}}}
$$


which sends 1 to $\left(f^{\left\lfloor t\left(p^{d}-1\right)\right\rfloor}\right)^{\frac{1}{p^{d}}}$. Choose $e>d$ such that

$$
\left(f^{\left\lceil t\left(p^{e}-1\right)\right\rceil}\right)^{\frac{1}{p^{e}}} F^{e}: R \rightarrow R^{\frac{1}{p^{e}}}
$$

splits. Since $R$ itself is $F$-split, we can compose the map $\left(f^{\left\lfloor t\left(p^{d}-1\right)\right\rfloor}\right)^{\frac{1}{p^{d}}} F^{d}$ with $F^{e-d}$.

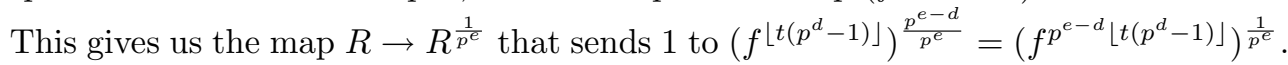
Thus it is sufficient to show that $p^{e-d}\left\lfloor t\left(p^{d}-1\right)\right\rfloor \leq\left\lceil t\left(p^{e}-1\right)\right\rceil$. Of course,

$$
p^{e-d}\left\lfloor t\left(p^{d}-1\right)\right\rfloor \leq p^{e-d}\left(t\left(p^{d}-1\right)\right)=t\left(p^{e}-p^{e-d}\right) \leq t\left(p^{e}-1\right) \leq\left\lceil t\left(p^{e}-1\right)\right\rceil
$$

as desired.

Corollary 3.6. If there is an $f \in R$ such that the map $f^{\frac{1}{p^{e}}} F^{e}: R \rightarrow R^{\frac{1}{p^{e}}}$ splits, then $\left(R, f^{\frac{1}{p^{e}-1}}\right)$ is F-pure.

Corollary 3.7. The pair $\left(R, \mathfrak{a}^{t}\right)$ is sharply $F$-pure if and only if there exists some $e>0$ and $f \in \mathfrak{a}^{\left\lceil t\left(p^{e}-1\right)\right\rceil}$ such that $\left(R, f^{\frac{1}{p^{e}-1}}\right)$ is F-pure (equivalently, $\left(R, f^{\frac{1}{p^{e}-1}}\right)$ is sharply F-pure).

Proof. If $\left(R, \mathfrak{a}^{t}\right)$ is sharply $F$-pure, then it follows by Corollary 3.4 that there exists some $e$ and $f$ as above such that $\left(R, f^{\frac{1}{p^{e}-1}}\right)$ is sharply $F$-pure, and so it follows, by Proposition 3.5, that $\left(R, f^{\frac{1}{p^{e}-1}}\right)$ is $F$-pure.

Conversely, if there exists an $e$ and $f$ as above such that $\left(R, f^{\frac{1}{p^{e}-1}}\right)$ is $F$-pure, then the pair is also sharply F-pure by Remark 3.2. Furthermore, by Proposition 3.5, the map

$$
\left(f^{\left\lfloor\frac{1}{p^{e}-1}\left(p^{e}-1\right)\right\rfloor}\right)^{\frac{1}{p^{e}}} F^{e}: R \rightarrow R^{\frac{1}{p^{e}}}
$$

splits. But $f^{\left\lfloor\frac{1}{p^{e}-1}\left(p^{e}-1\right)\right\rfloor}=f \in \mathfrak{a}^{\left\lceil t\left(p^{e}-1\right)\right\rceil}$ by assumption, and so the corollary is proven.

The previous results suggest the following simple question.

Question 3.8. If $\left(R, \mathfrak{a}^{t}\right)$ is sharply $F$-pure, then is $\left(R, \mathfrak{a}^{t}\right) F$-pure?

Remark 3.9. In Section 5, we show that if $\left(R, \mathfrak{a}^{t}\right)$ is sharply $F$-pure, then $\left(R, \mathfrak{a}^{t-\epsilon}\right)$ is strongly $F$-pure (and thus $F$-pure) for every $\epsilon$ satisfying $t \geq \epsilon>0$.

Definition 3.10. Given an ideal $I \subseteq R$, we define the $\mathfrak{a}^{t}$-sharp Frobenius closure of $I$, denoted $I^{F^{\sharp} \mathfrak{a}^{t}}$, as follows. The ideal $I^{F^{\sharp} \mathfrak{a}^{t}}$ is the set of all elements $z$ such that $\mathfrak{a}^{\left[t\left(p^{e}-1\right)\right\rceil} z^{p^{e}} \subseteq I^{\left[p^{e}\right]}$ for all $e \gg 0$.

Remark 3.11. Note that if $\left(R, \mathfrak{a}^{t}\right)$ is sharply $F$-pure, then $I^{F^{\sharp} \mathfrak{a}^{t}}=I$ for all ideals $I$. The argument is essentially the same as the argument that a strongly $F$-regular ring is weakly $F$-regular; see [8, Proposition 3.1].

Remark 3.12. In Definition 3.10, it might seem natural to replace the condition "for all $e \gg 0$ " with the condition "for infinitely many $e>0$ ". We will be working in the case of an sharply $F$-pure pair. Therefore, if for infinitely many $e>0$, we have the containment $\mathfrak{a}^{\left\lceil t\left(p^{e}-1\right)\right\rceil} z^{p^{e}} \subseteq I^{\left[p^{e}\right]}$, it is not clear whether we still have such a containment at an $e>0$ where the map used to define sharp $F$-purity splits. Furthermore, the condition that $\mathfrak{a}^{\left[t\left(p^{e}-1\right)\right]} z^{p^{e}} \subseteq I^{\left[p^{e}\right]}$ for all $e \gg 0$ arises naturally in Lemma 3.13.

The following lemma can be used to check containment in common situations. 
Lemma 3.13. In the setup of the previous definition, suppose that $\mathfrak{a}=(f)$ and there exists some $e>0$ such that $\mathfrak{a}^{\left[t\left(p^{e}-1\right)\right]} z^{p^{e}} \subseteq I^{\left[p^{e}\right]}$ and so that $t\left(p^{e}-1\right)$ is an integer, then $z \in I^{F^{\sharp} \mathfrak{a}^{t}}$

Proof. Suppose that $f^{\left[t\left(p^{e}-1\right)\right\rceil} z^{p^{e}} \subseteq I^{\left[p^{e}\right]}$. Then, for any integer $d>0$ we have

$$
f^{p^{d}\left\lceil t\left(p^{e}-1\right)\right\rceil} z^{p^{e+d}} \subseteq I^{\left[p^{e+d}\right]} .
$$

Therefore, it suffices to show that $\left\lceil t\left(p^{d+e}-1\right)\right\rceil \geq p^{d}\left\lceil t\left(p^{e}-1\right)\right\rceil$. Of course $t\left(p^{e}-1\right)=$ $\left\lceil t\left(p^{e}-1\right)\right\rceil$ by assumption so that $p^{d}\left\lceil t\left(p^{e}-1\right)\right\rceil=p^{d} t\left(p^{e}-1\right)=p^{d+e} t-t p^{d}$. Now, note that $\left\lceil t\left(p^{d+e}-1\right)\right\rceil \geq t\left(p^{d+e}-1\right) \geq p^{d+e} t-t p^{d}$.

Our last goal of the section is to prove that sharply $F$-pure pairs have radical test ideals.

Proposition 3.14. Suppose $c$ is an element of $R$ such that some power of $c$ is contained in $\tau\left(\mathfrak{a}^{t}\right)$. Then for all ideals $I$, we have $c I^{* \mathfrak{a}^{t}} \subseteq I^{F^{\sharp} \mathfrak{a}^{t}}$.

Proof. Choose $z \in I^{* \mathfrak{a}^{t}}$. Consider all $e \geq e_{0}$ such that $c^{p^{e}}$ is in $\tau\left(\mathfrak{a}^{t}\right)$. But then we know that $\mathfrak{a}^{\left[t\left(p^{e}-1\right)\right\rceil}(c z)^{p^{e}}=c^{p^{e}} \mathfrak{a}^{\left[t\left(p^{e}-1\right)\right\rceil} z^{p^{e}} \subseteq I^{\left[p^{e}\right]}$ for all $e \geq e_{0}$. This implies that $c z \in I^{F^{\sharp} \mathfrak{a}^{t}}$.

Corollary 3.15. Suppose that $\left(R, \mathfrak{a}^{t}\right)$ is sharply F-pure. Then $\tau\left(\mathfrak{a}^{t}\right)$ is a radical ideal.

Proof. Suppose that $c^{n} \in \tau\left(\mathfrak{a}^{t}\right)$ for some $n>0$. Then we know that for any ideal $I$, $c I^{* \mathfrak{a}^{t}} \subseteq I^{F^{\sharp} \mathfrak{a}^{t}}=I$ where the final equality comes because $\left(R, \mathfrak{a}^{t}\right)$ is sharply $F$-pure. Thus $c \in \tau\left(\mathfrak{a}^{t}\right)$ as desired.

Corollary 3.16. Suppose that $\left(R, \mathfrak{a}^{t}\right)$ is F-pure and that $\left(p^{e}-1\right) t$ is an integer for some (equivalently infinitely many) $e>0$. Then $\tau\left(\mathfrak{a}^{t}\right)$ is a radical ideal.

If instead of sharp $F$-purity one considers the standard definition ( $F$-purity), then Yoshida and Mustață have constructed examples of pairs $\left(R, f^{t}\right)$ that are $F$-pure, but which have non-radical test-ideals; see [15, Example 4.5].

\section{On a generalization of a result of Vassilev's}

The first goal of this section is to show that a Fedder-type criterion extends to the context of sharply $F$-pure pairs. As an application we show that the main result of [21], also extends to the context of sharply $F$-pure pairs. Her result, and its generalization below, can be thought of as closely related to certain subadjunctiontype results in characteristic zero such as [1], [12] and [16, Theorem 5.5].

Theorem 4.1. [3, Proposition 1.7], [6, Proposition 2.6], [18, Lemmas 3.4, 3.9] Suppose $(S, \mathfrak{m})$ is an $F$-finite regular local ring, $I \subseteq S$ a radical ideal, $R=S / I, \mathfrak{a}^{\prime}$ an ideal of $S$ containing $I$, with image in $R$ denoted by $\mathfrak{a}$ such that $\mathfrak{a} \cap R^{\circ} \neq \emptyset$, and $t>0$ is a real number. Then the following are equivalent.

(i) $\left(R, \mathfrak{a}^{t}\right)$ is sharply F-pure,

(ii) For infinitely many $q=p^{e}$, there exists $d \in \mathfrak{a}^{\lceil t(q-1)\rceil}$ such that $d^{\frac{1}{p^{e}}} F^{e}: E_{R} \rightarrow$ $E_{R} \otimes_{R} R^{\frac{1}{q}}$ is injective,

(iii) $\mathfrak{a}^{\prime[t(q-1)]}\left(I^{[q]}: I\right) \nsubseteq \mathfrak{m}^{[q]}$ for infinitely many $q=p^{e}$. 
Proof. We begin by proving the equivalence of (i) and (ii). Note that for any $d \in R$, the map $d^{\frac{1}{q}} F^{e}: R \rightarrow R^{\frac{1}{q}}$ splits if and only if the composition

$$
\operatorname{Hom}_{R}\left(R^{\frac{1}{q}}, R\right) \stackrel{d^{\frac{1}{q}}}{\longrightarrow} \operatorname{Hom}_{R}\left(R^{\frac{1}{q}}, R\right) \rightarrow \operatorname{Hom}_{R}(R, R)
$$

is surjective. This surjection is clearly invariant under completion, so we may assume that $R$ is complete. Following [18, Lemma 3.4], we wish to show that the Matlis dual of $\operatorname{Hom}_{R}\left(R^{\frac{1}{q}}, R\right)$ is isomorphic to $E_{R} \otimes_{R} R^{\frac{1}{q}}$. But note that, by Matlis duality, $\operatorname{Hom}_{R}\left(R^{\frac{1}{q}}, R\right) \cong \operatorname{Hom}_{R}\left(\operatorname{Hom}_{R}\left(R, E_{R}\right), \operatorname{Hom}_{R}\left(R^{\frac{1}{q}}, E_{R}\right)\right)$. On the other hand, the right side is naturally isomorphic to $\operatorname{Hom}_{R}\left(E_{R} \otimes_{R} R^{\frac{1}{q}}, E_{R}\right)$ which is precisely the Matlis dual of $E_{R} \otimes_{R} R^{\frac{1}{q}}$ as desired. But then we see, by Matlis duality, that the map 4.1.1 is surjective if and only if $d^{\frac{1}{p^{e}}} F^{e}: E_{R} \rightarrow E_{R} \otimes_{R} R^{\frac{1}{q}}$ is injective.

Instead of proving the equivalence of (ii) and (iii) we simply refer to the proof of [18, Theorem 3.9]. In that proof, Takagi shows that $d^{\frac{1}{p^{e}}} F^{e}: E_{R} \rightarrow E_{R} \otimes_{R} R^{\frac{1}{p^{e}}}$ injects if and only if $d\left(I^{\left[p^{e}\right]}: I\right) \nsubseteq \mathfrak{m}^{\left[p^{e}\right]}$, which is precisely what we need.

Theorem 4.2. [21, Theorem 3.1] Let $S$ be an $F$-finite regular local ring and $I \subseteq S$ a radical ideal. Suppose that $\mathfrak{a} \subseteq S$ is an ideal containing $I$ and $t>0$ is a real number. Let us denote $S / I$ by $R$ and $\mathfrak{a} / I$ by $\overline{\mathfrak{a}}$. If $\phi: S \rightarrow R$ is the canonical surjection, then $\mathfrak{a}^{[t(q-1)\rceil}\left(I^{[q]}: I\right) \subseteq\left(\left(\phi^{-1}\left(\tau_{R}\left(\overline{\mathfrak{a}}^{t}\right)\right)\right)^{[q]}: \phi^{-1}\left(\tau_{R}\left(\overline{\mathfrak{a}}^{t}\right)\right)\right)$ for all $q=p^{e}>0$.

Proof. The proof is a straightforward generalization of Vassilev's proof of the same statement in the case that $\mathfrak{a}=R$. Given $J \subseteq S$ which contains $I$, let us denote $J / I \subseteq R$ by $\bar{J}$. Notice that for all $\bar{J} \subseteq R$, all $q=p^{e}$, all $c \in \tau_{R}\left(\overline{\mathfrak{a}}^{t}\right)$ and all $z \in \bar{J}^{\overline{\mathfrak{a}}^{t}}$ we have $c \overline{\mathfrak{a}}^{[t(q-1)\rceil} z^{q} \subseteq \bar{J}^{[q]}$. In other words, we have

$$
\left(\tau_{R}\left(\overline{\mathfrak{a}}^{t}\right)\right) \overline{\mathfrak{a}}^{[t(q-1)\rceil}\left(\left(\bar{J}^{* \mathfrak{a}^{t}}\right)^{[q]}\right) \subseteq \bar{J}^{[q]} .
$$

Pulling this back to $S$, we obtain

$$
\phi^{-1}\left(\tau_{R}\left(\overline{\mathfrak{a}}^{t}\right)\right) \mathfrak{a}^{[t(q-1)\rceil}\left(\phi^{-1}\left(\bar{J}^{* \overline{\mathfrak{a}}^{t}}\right)\right)^{[q]} \subseteq \phi^{-1}\left(\tau_{R}\left(\overline{\mathfrak{a}}^{t}\right)\right) \phi^{-1}\left(\overline{\mathfrak{a}}^{[t(q-1)\rceil}\right)\left(\phi^{-1}\left(\left(\bar{J}^{* \overline{\mathfrak{a}}^{t}}\right)^{[q]}\right)\right) \subseteq J^{[q]}+I .
$$

Choose $w \in\left(I^{[q]}: I\right)$ and note that it is sufficient to show that $w \mathfrak{a}^{\lceil t(q-1)\rceil} \subseteq$ $\left(\left(\phi^{-1}\left(\tau_{R}\left(\overline{\mathfrak{a}}^{t}\right)\right)\right)^{[q]}: \phi^{-1}\left(\tau_{R}\left(\overline{\mathfrak{a}}^{t}\right)\right)\right)$. Observe that

$$
w \phi^{-1}\left(\tau_{R}\left(\overline{\mathfrak{a}}^{t}\right)\right) \mathfrak{a}^{[t(q-1)\rceil}\left(\phi^{-1}\left(\bar{J}^{* \overline{\mathfrak{a}}^{t}}\right)\right)^{[q]} \subseteq w J^{[q]}+I^{[q]} \subseteq J^{[q]} .
$$

Therefore,

$$
w \phi^{-1}\left(\tau_{R}\left(\overline{\mathfrak{a}}^{t}\right)\right) \mathfrak{a}^{[t(q-1)]} \subseteq\left(J^{[q]}:\left(\phi^{-1}\left(\bar{J}^{* \overline{\mathfrak{a}}^{t}}\right)\right)^{[q]}\right)=\left(J:\left(\phi^{-1}\left(\bar{J}^{* \overline{\mathfrak{a}}^{t}}\right)\right)\right)^{[q]} .
$$

But this implies that $w \phi^{-1}\left(\tau_{R}\left(\overline{\mathfrak{a}}^{t}\right)\right) \mathfrak{a}^{\lceil t(q-1)]} \subseteq \bigcap_{I \subseteq J}\left(J:\left(\phi^{-1}\left(\bar{J}^{* \overline{\mathfrak{a}}^{t}}\right)\right)\right)^{[q]}$. By $[21$, Lemma 2.1], we see that $w \mathfrak{a}^{\lceil t(q-1)\rceil} \phi^{-1}\left(\tau_{R}\left(\overline{\mathfrak{a}}^{t}\right)\right) \subseteq\left(\bigcap_{I \subseteq J}\left(J:\left(\phi^{-1}\left(\bar{J}^{* \overline{\mathfrak{a}}^{t}}\right)\right)\right)\right)^{[q]}$.

Thus, we simply have to prove that $\bigcap_{I \subseteq J}\left(J:\left(\phi^{-1}\left(\bar{J}^{\overline{\mathfrak{a}}^{t}}\right)\right)\right)=\phi^{-1}\left(\tau_{R}\left(\overline{\mathfrak{a}}^{t}\right)\right)$, and we will be done. Therefore, we consider

$$
v \in \bigcap_{I \subseteq J}\left(J:\left(\phi^{-1}\left(\bar{J}^{* \mathfrak{a}^{t}}\right)\right)\right) .
$$


This condition on $v$ is equivalent to requiring that $v \phi^{-1}\left(\bar{J}^{\overline{\mathfrak{a}}^{t}}\right) \subseteq J$ for all $\bar{J} \subseteq R$. Of course, this is the same as the condition $\phi(v) \bar{J}^{\overline{\mathfrak{a}}^{t}} \subseteq \bar{J}$, or in other words $\phi(v) \in$ $\left(\bar{J}: \bar{J}^{\overline{\mathfrak{a}}^{t}}\right)$ for all $\bar{J} \subseteq R$. This last statement is equivalent to $v \in \phi^{-1}\left(\tau_{R}\left(\overline{\mathfrak{a}}^{t}\right)\right)$ as desired.

Corollary 4.3. Suppose $R$ is a quotient of an $F$-finite regular ring, $\mathfrak{a} \subseteq R$ an ideal such that $\mathfrak{a} \cap R^{\circ} \neq \emptyset$ and $t>0$ a real number such that $\left(R, \mathfrak{a}^{t}\right)$ is sharply F-pure. Then $R / \tau_{R}\left(\mathfrak{a}^{t}\right)$ is F-pure.

\section{An application to $F$-pure thresholds}

We begin by recalling the definition of the $F$-pure threshold. As before, we assume that $R$ is an $F$-finite reduced ring and that $\mathfrak{a} \cap R^{\circ} \neq \emptyset$.

Definition 5.1. [20, Definition 2.1] [20, Proposition 2.2(3)] Let $R$ be a reduced $F$ finite ring and $\mathfrak{a} \subseteq R$ an ideal such that $\mathfrak{a} \cap R^{\circ} \neq \emptyset$. We define the $F$-pure threshold, $\mathrm{c}(\mathfrak{a})$ to be

$\mathrm{c}(\mathfrak{a})=\sup \left\{s \in \mathbb{R}_{\geq 0} \mid\left(R, \mathfrak{a}^{s}\right)\right.$ is $F$-pure $\}=\sup \left\{s \in \mathbb{R}_{\geq 0} \mid\left(R, \mathfrak{a}^{s}\right)\right.$ is strongly $F$-pure $\}$.

Our next goal is to show that the $F$-pure threshold can be described using sharp $F$-purity. First we need a lemma.

Lemma 5.2. If $\left(R, \mathfrak{a}^{s}\right)$ is sharply $F$-pure, then $\left(R, \mathfrak{a}^{s-\epsilon}\right)$ is strongly $F$-pure (and thus also $F$-pure) for all $\epsilon$ satisfying $0<\epsilon \leq s$.

Proof. Choose $e>0$ that satisfies $\epsilon p^{e}>s$ and such that there exists an $f \in \mathfrak{a}^{\left\lceil s\left(p^{e}-1\right)\right\rceil}$ so that the map $f^{\frac{1}{p^{e}}} F^{e}$ splits. Then $s\left(p^{e}-1\right)>p^{e}(s-\epsilon)$ and so $\left\lceil s\left(p^{e}-1\right)\right\rceil \geq\left\lceil p^{e}(s-\epsilon)\right\rceil$ which implies that

$$
f \in \mathfrak{a}^{\left\lceil s\left(p^{e}-1\right)\right\rceil} \subseteq \mathfrak{a}^{\left\lceil(s-\epsilon)\left(p^{e}\right)\right\rceil} .
$$

But then we see that $\left(R, \mathfrak{a}^{s-\epsilon}\right)$ is strongly $F$-pure as desired.

Proposition 5.3. Let $R$ and $\mathfrak{a}$ be as above then,

$$
\mathrm{c}(\mathfrak{a})=\sup \left\{s \in \mathbb{R}_{\geq 0} \mid \text { the pair }\left(R, \mathfrak{a}^{s}\right) \text { is sharply F-pure }\right\} .
$$

Proof. First note that a strongly $F$-pure pair is always sharply $F$-pure, so we see that the inequality $\leq$ is easy. The other inequality is immediately implied by Lemma 5.2 .

Using this, we can show that the $F$-pure threshold is a certain type of rational number under certain conditions; compare with [2], [11], or [4].

Corollary 5.4. Suppose that $\left(R, \mathfrak{a}^{\mathrm{c}(\mathfrak{a})}\right)$ is a sharply F-pure pair. Then $\mathrm{c}(\mathfrak{a})$ is a rational number and, furthermore, there exists some $e>0$ such that $\mathrm{c}(\mathfrak{a})\left(p^{e}-1\right) \in \mathbb{Z}$. In other words, $\mathrm{c}(\mathfrak{a})$ can be written as a quotient $\frac{m}{n}$ where $m, n \in \mathbb{Z}$ and $p$ does not divide $n$.

Proof. Assume that $\mathrm{c}(\mathfrak{a})$ cannot be written as such a quotient. Now suppose $\left(R, \mathfrak{a}^{\mathrm{c}(\mathfrak{a})}\right)$ is sharply $F$-pure, then there exists an $f \in \mathfrak{a}^{\left\lceil\mathrm{c}(\mathfrak{a})\left(p^{e}-1\right)\right\rceil}$ such that $f^{\frac{1}{p^{e}}} F^{e}: R \rightarrow R^{\frac{1}{p^{e}}}$ splits. But then note that $f \in \mathfrak{a}^{\left\lceil(\mathrm{c}(\mathfrak{a})+\epsilon)\left(p^{e}-1\right)\right\rceil}$ for some sufficiently small $\epsilon>0$, since $\mathrm{c}(\mathfrak{a})\left(p^{e}-1\right)$ is not an integer. This contradicts Proposition 5.3. 
Remark 5.5. Mircea Mustaţă has suggested the following argument which gives a partial converse to Corollary 5.4. Consider the situation where $(R, \mathfrak{m})$ is an $F$-finite regular local ring and $\mathfrak{a}=(f)$ is principal. Suppose that $\mathrm{c}(f)$ is a rational number such that $\mathrm{c}(f)\left(p^{e}-1\right) \in \mathbb{Z}$ for some $e>0$ and $\mathrm{c}(f)<1$. We will sketch Mustaţă's argument that $\left(R, f^{c(f)}\right)$ is (sharply) $F$-pure.

Following the notation of [14], we set $\nu_{f}\left(p^{e}\right)=\max \left\{s \in \mathbb{Z}_{>0} \mid f^{s} \notin \mathfrak{m}^{\left[p^{e}\right]}\right\}$. Fix an $e>0$ and note that $p^{d} \nu_{f}\left(p^{e}\right) \leq \nu_{f}\left(p^{e+d}\right) \leq p^{d}\left(\nu_{f}\left(p^{e}\right)+1\right)$ since $f^{r} \in \mathfrak{m}^{\left[p^{e}\right]}$ if and only if $f^{r p^{d}} \in \mathfrak{m}^{\left[p^{e+d}\right]}$. Dividing through by $p^{d+e}$ gives us

$$
\frac{\nu_{f}\left(p^{e}\right)}{p^{e}} \leq \frac{\nu_{f}\left(p^{e+d}\right)}{p^{e+d}} \leq \frac{\nu_{f}\left(p^{e}\right)+1}{p^{e}} .
$$

We then take the limit as $d$ goes to infinity and obtain, by [14, Remark 1.5], that $\frac{\nu_{f}\left(p^{e}\right)}{p^{e}} \leq \mathrm{c}(f) \leq \frac{\nu_{f}\left(p^{e}\right)+1}{p^{e}}$, for every $e>0$. Now multiplying through by $p^{e}$ we get

$$
\nu_{f}\left(p^{e}\right) \leq p^{e} c(f) \leq \nu_{f}\left(p^{e}\right)+1
$$

for every $e>0$. Note that both inequalities are actually strict since under our hypotheses, $p^{e} \mathrm{c}(f)$ is never an integer.

Fix an $e>0$ such that $\left(p^{e}-1\right) \mathrm{c}(f)$ is an integer. To show that $\left(R, f^{\mathrm{c}(f)}\right)$ sharply $F$ pure, it is sufficient to show that $\nu_{f}\left(p^{e}\right)=\left\lceil\mathrm{c}(f)\left(p^{e}-1\right)\right\rceil=\mathrm{c}(f)\left(p^{e}-1\right)$ for some $e>0$ by Theorem 4.1. First observe that the fractional part $\left\{\mathrm{c}(f) p^{e}\right\}=\mathrm{c}(f) p^{e}-\left\lfloor\mathrm{c}(f) p^{e}\right\rfloor$ is equal to $\mathrm{c}(f)$ because $\left\{\mathrm{c}(f) p^{e}\right\}=\left\{\mathrm{c}(f)\left(p^{e}-1\right)+\mathrm{c}(f)\right\}=\{\mathrm{c}(f)\}$. By rounding down equation 5.5.1, we see that

$$
\nu_{f}\left(p^{e}\right)=\left\lfloor\mathrm{c}(f) p^{e}\right\rfloor=\mathrm{c}(f) p^{e}-\mathrm{c}(f)=\mathrm{c}(f)\left(p^{e}-1\right)<\nu_{f}\left(p^{e}\right)+1,
$$

which completes the argument.

We conclude with one more application of Lemma 5.2.

Corollary 5.6. If $\left(R, \mathfrak{a}^{t}\right)$ has dense sharply F-pure type and $X=\operatorname{Spec} R$ is normal and $\mathbb{Q}$-Gorenstein, then $\left(X, \mathfrak{a}^{t}\right)$ is log canonical.

For a definition of log canonical singularities see [13]. By dense sharply F-pure type we simply mean to generalize the usual definition of dense F-pure type; see, for example, [20, Definition 1.9], by replacing the phrase " $F$-pure" wherever it occurs, with "sharply F-pure". Roughly, dense sharply F-pure type means that, after reducing the pair to a collection of positive characteristic models, a Zariski dense set of those models is sharply $F$-pure. In order to prove Corollary 5.6, one could repeat the argument of [6, Theorem 3.3] or [18, Theorem 3.8]. We instead use Lemma 5.2 and apply [18, Theorem 3.8].

Proof. It is easy to see that $\left(X, \mathfrak{a}^{t}\right)$ is $\log$ canonical if and only if $\left(X, \mathfrak{a}^{t-\epsilon}\right)$ is $\log$ canonical for every $\epsilon$ satisfying $t>\epsilon>0$. But note that if $\left(R, \mathfrak{a}^{t}\right)$ has dense sharply $F$-pure type, then $\left(R, \mathfrak{a}^{t-\epsilon}\right)$ has dense $F$-pure type by Proposition 5.2. But then by [18, Theorem 3.8], $\left(X, \mathfrak{a}^{t-\epsilon}\right)$ is $\log$ canonical.

Remark 5.7. Suppose that $\left(R, \mathfrak{a}^{t}\right)$ has dense $F$-pure type and $t=a / b$ is a rational number where $a$ and $b$ are integers. Further suppose that $p$ is a prime that doesn't appear as a factor of $b$ and note that all but a finite number of primes satisfy this property. Therefore, for infinitely many $e>0, t\left(p^{e}-1\right)$ is an integer. It thus follows that $\left(R, \mathfrak{a}^{t}\right)$ has dense sharply $F$-pure type. 


\section{Acknowledgements}

The author would like to thank Mircea Mustaţă and Mel Hochster for several valuable discussions, thank Shunsuke Takagi for pointing out several typos in a previous draft, and also thank Mel Hochster for a careful reading of this paper. Finally, the author would like to thank the referee for several valuable suggestions as well as pointing out several typos.

\section{References}

[1] F. Ambro, The locus of log canonical singularities, Preprint available at arXiv:math.AG/9806067 (1998).

[2] M. Blickle, M. Mustaţă, and K. Smith, Discreteness and rationality of F-thresholds, To appear in Trans. Amer. Math. Soc. (2006).

[3] R. Fedder, F-purity and rational singularity, Trans. Amer. Math. Soc. 278 (1983), no. 2, 461480.

[4] N. Hara, F-pure thresholds and F-jumping exponents in dimension two, Math. Res. Lett. 13 (2006), no. 5-6, 747-760. With an appendix by Paul Monsky.

[5] N. Hara and S. Takagi, On a generalization of test ideals, Nagoya Math. J. 175 (2004) 59-74.

[6] N. Hara and K.-I. Watanabe, F-regular and F-pure rings vs. log terminal and log canonical singularities, J. Algebraic Geom. 11 (2002), no. 2, 363-392.

[7] N. Hara and K.-I. Yoshida, A generalization of tight closure and multiplier ideals, Trans. Amer. Math. Soc. 355 (2003), no. 8, 3143-3174 (electronic).

[8] M. Hochster and C. Huneke, Tight closure and strong F-regularity, Mém. Soc. Math. France (N.S.) (1989), no. 38, 119-133. Colloque en l'honneur de Pierre Samuel (Orsay, 1987).

[9] - Tight closure, invariant theory, and the Briançon-Skoda theorem, J. Amer. Math. Soc. 3 (1990), no. 1, 31-116.

[10] M. Hochster and J. L. Roberts, The purity of the Frobenius and local cohomology, Advances in Math. 21 (1976), no. 2, 117-172.

[11] M. Katzman, G. Lyubeznik, and W. Zhang, On the discreteness and rationality of jumping coefficients (2007), Preprint available at arXiv:0706.3028.

[12] Y. Kawamata, Subadjunction of log canonical divisors. II, Amer. J. Math. 120 (1998), no. 5, 893-899.

[13] J. Kollár and S. Mori, Birational geometry of algebraic varieties, Vol. 134 of Cambridge Tracts in Mathematics, Cambridge University Press, Cambridge (1998), ISBN 0-521-63277-3. With the collaboration of C. H. Clemens and A. Corti, Translated from the 1998 Japanese original.

[14] M. Mustaţă, S. Takagi, and K.-i. Watanabe, F-thresholds and Bernstein-Sato polynomials, in European Congress of Mathematics, 341-364, Eur. Math. Soc., Zürich (2005).

[15] M. Mustaţă and K.-I. Yoshida, Test ideals vs. multiplier ideals To appear in Nagoya Math. J. (2007).

[16] K. Schwede, A simple characterization of Du Bois singularities, Compos. Math. 143 (2007), no. $4,813-828$.

[17] K. E. Smith, The multiplier ideal is a universal test ideal, Comm. Algebra 28 (2000), no. 12, 5915-5929. Special issue in honor of Robin Hartshorne.

[18] S. Takagi, F-singularities of pairs and inversion of adjunction of arbitrary codimension, Invent. Math. 157 (2004), no. 1, 123-146.

[19] — An interpretation of multiplier ideals via tight closure, J. Algebraic Geom. 13 (2004), no. $2,393-415$

[20] S. Takagi and K.-i. Watanabe, On F-pure thresholds, J. Algebra 282 (2004), no. 1, 278-297.

[21] J. C. Vassilev, Test ideals in quotients of F-finite regular local rings, Trans. Amer. Math. Soc. 350 (1998), no. 10, 4041-4051.

Department of Mathematics, University of Michigan, East Hall, 530 Church Street, Ann Arbor, Michigan, 48109

E-mail address: kschwede@umich.edu 